\title{
A Generation Result for Cosine Functions of Operators
}

\author{
Hernán R. Henríquez and Carlos Landero H.
}

\begin{abstract}
In this work a generation result for strongly continuous cosine functions of operators is established, and the result is applied to study a geometric property of self-accessible states of linear second order abstract control systems.
\end{abstract}

Keywords. Cosine functions of operators, control systems of second order Mathematics Subject Classification (2000). Primary 47D09, secondary 34G10, 93B05

\section{Introduction.}

Throughout this work $X$ denotes a Banach space endowed with a norm $\|\cdot\|$ and $A: D(A) \subseteq X \rightarrow X$ is a closed linear operator. In [3] a characterization of a well posed first order abstract Cauchy problem (abbreviated by ACP)

$$
\begin{aligned}
& x^{\prime}(t)=A x(t)+f(t), \quad t \in I=[0, a], \\
& x(0)=x_{0} \in X
\end{aligned}
$$

was established, where $f: I \rightarrow X$ is an integrable function. The aim of this paper is to show that a similar result holds for the second order ACP

$$
\begin{aligned}
x^{\prime \prime}(t) & =A x(t)+f(t), \quad t \in I=[0, a], \\
x(0) & =x_{0}, \quad x^{\prime}(0)=x_{1} .
\end{aligned}
$$

As an application we study a geometric property of self-accessible trajectories of second order distributed control systems. Our exposition is based on the theory of strongly continuous cosine functions of linear operators. In this section we review some fundamental aspects of this theory needed to establish our results.

H. R. Henríquez: Departamento de Matemática, Universidad de Santiago, USACH, Casilla 307, Correo-2, Santiago, Chile; hernan.henriquez@usach.cl

C. Landero H.: Instituto de Ciencias Básicas, Universidad Diego Portales, Casilla 298-V, Santiago, Chile; landerocarlos@gmail.com

This work was supported in part by FONDECYT, Grant 1050314. 
The terminology and notations are those generally used in functional analysis. In particular, if $\left(Z,\|\cdot\|_{Z}\right)$ and $\left(Y,\|\cdot\|_{Y}\right)$ are Banach spaces, we indicate by $\mathcal{L}(Z, Y)$ the Banach space of bounded linear operators from $Z$ into $Y$ endowed with the uniform operator topology. We abbreviate this notation to $\mathcal{L}(Z)$ whenever $Z=Y$, and $Z^{*}=\mathcal{L}(Z, \mathbb{K})$ denotes the dual space of $Z$. In addition, if $T$ is a linear operator, then $\mathcal{R}(T)$ designates the range space of $T$, and if $T$ is a linear operator defined in a dense subspace $D(T)$ of $Z$ we represent by $T^{*}$ the adjoint of $T$. Finally, along this paper, $C(I, Z)$ is the space of continuous functions from $I$ into $Z$ endowed with the norm of the uniform convergence.

For the theory of cosine functions of operators we refer to $[1,5,8,20]$. Next we only mention a few concepts and properties relative to the second order abstract Cauchy problem. A function $C:(-\infty, \infty) \rightarrow \mathcal{L}(X)$ is called a strongly continuous cosine function of operators if the following conditions hold:

(i) $C(0)=I$;

(ii) $C(t+s)+C(t-s)=2 C(t) C(s)$ for all $s, t \in(-\infty, \infty)$;

(iii) for each $x \in X$, the function $(-\infty, \infty) \rightarrow X, t \mapsto C(t) x$, is continuous.

The infinitesimal generator $A: D(A) \subseteq X \rightarrow X$ of $C(t)$ is defined by

$$
A x=2 \lim _{h \rightarrow 0} \frac{C(h) x-x}{h^{2}}, \quad x \in D(A),
$$

and the domain of $A$ is the subspace consisting of all $x \in X$ such that the preceding limit exists. We denote by $S(t)$ the sine function associated to $C(t)$, which is defined by

$$
S(t) x=\int_{0}^{t} C(s) x d s, \quad x \in X, t \in \mathbb{R} .
$$

The notation $E$ stands for the space formed by the vectors $x \in X$ for which $C(\cdot) x$ is of class $C^{1}$ on $\mathbb{R}$. We know from Kisińsky [14], that $E$ endowed with the norm

$$
\|x\|_{E}=\|x\|+\sup _{0 \leq t \leq 1}\|A S(t) x\|, \quad x \in E,
$$

is a Banach space. The operator valued function $G(t)=\left[\begin{array}{cc}C(t) & S(t) \\ A S(t) & C(t)\end{array}\right]$ is a strongly continuous group of bounded linear operators on the space $E \times X$, generated by the operator $\mathcal{A}=\left[\begin{array}{ll}0 & I \\ A & 0\end{array}\right]$ defined on $D(A) \times E$. It follows from this that $S(t)(E) \subseteq D(A)$, the linear operator $A S(t): E \rightarrow X$ is bounded and $A S(t) x \rightarrow 0$, $t \rightarrow 0$, for each $x \in E$. Furthermore, if $x:[0, \infty) \rightarrow X$ is locally integrable, then $y(t)=\int_{0}^{t} S(t-s) x(s) d s$ defines an $E$-valued continuous function, which is a consequence of the fact that

$$
\int_{0}^{t} G(t-s)\left[\begin{array}{c}
0 \\
x(s)
\end{array}\right] d s=\left[\begin{array}{c}
\int_{0}^{t} S(t-s) x(s) d s \\
\int_{0}^{t} C(t-s) x(s) d s
\end{array}\right]
$$

defines an $E \times X$-valued continuous function. 
The existence of solutions for the second order abstract Cauchy problem (1.1)-(1.2) has been discussed in [18]. Similarly, the existence of solutions for the semilinear second order abstract Cauchy problem has been treated in [19]. Let $f$ be a function in $\mathcal{L}^{1}(I, X)$. We only mention here that the function $u(\cdot)$ given by

$$
u(t)=C(t) x_{0}+S(t) x_{1}+\int_{0}^{t} S(t-s) f(s) d s, \quad t \in I,
$$

is called mild solution of $(1.1)-(1.2)$. When $x_{0} \in E$, this function $u(\cdot)$ is continuously differentiable and

$$
u^{\prime}(t)=A S(t) x_{0}+C(t) x_{1}+\int_{0}^{t} C(t-s) f(s) d s .
$$

The regularity of mild solutions of (1.1)-(1.2) has been treated in Travis \& Webb [19], Bochenek [4] and Henríquez \& Vásquez [12].

In the following lemma we collect some properties of cosine functions that will be needed in the next section.

Lemma 1.1. Let $A$ be the infinitesimal generator of a strongly continuous cosine function of operators $C$. Then the following properties are fulfilled:

(a) For every $x \in X$ and $t \in \mathbb{R}, \int_{0}^{t} S(\xi) x d \xi \in D(A)$ and

$$
A \int_{0}^{t} S(\xi) x d \xi=C(t) x-x
$$

(b) For every $f \in \mathcal{L}^{1}([0, \tau], X), \int_{0}^{\tau} \int_{0}^{t} S(t-\xi) f(\xi) d \xi d t \in D(A)$ and

$$
A \int_{0}^{\tau} \int_{0}^{t} S(t-\xi) f(\xi) d \xi d t=\int_{0}^{\tau}[C(\tau-s)-I] f(s) d s
$$

(c) Let $x^{*} \in D\left(A^{*}\right)$. Then the function $C(\cdot)^{*} x^{*}$ is continuously differentiable and

$$
\frac{d}{d t} C(t)^{*} x^{*}=S(t)^{*} A^{*} x^{*}
$$

(d) Let $x^{*} \in D\left(A^{*}\right)$. If $f \in \mathcal{L}^{1}([0, \tau], X)$, then the function $t \rightarrow \int_{0}^{t}\langle C(t-$ s) $\left.f(s), x^{*}\right\rangle d s$ is absolutely continuous and the equality

$$
\frac{d}{d t} \int_{0}^{t}\left\langle C(t-s) f(s), x^{*}\right\rangle d s=\left\langle f(t), x^{*}\right\rangle+\int_{0}^{t}\left\langle S(t-s) f(s), A^{*} x^{*}\right\rangle d s
$$

holds almost everywhere. 
Proof. Assertion (a) follows from [1, Proposition 3.14.5 (a)]. To prove (b), we observe that $\int_{0}^{\tau} \int_{0}^{t} S(t-\xi) f(\xi) d \xi d t=\int_{0}^{\tau} \int_{\xi}^{\tau} S(t-\xi) f(\xi) d t d \xi$. It follows from (a) that $\int_{\xi}^{\tau} S(t-\xi) f(\xi) d t \in D(A)$ and

$$
A \int_{\xi}^{\tau} S(t-\xi) f(\xi) d t=A \int_{0}^{\tau-\xi} S(s) f(\xi) d s=C(\tau-\xi) f(\xi)-f(\xi) .
$$

Since $A$ is a closed operator, we obtain

$$
A \int_{0}^{\tau} \int_{\xi}^{\tau} S(t-\xi) f(\xi) d t d \xi=\int_{0}^{\tau} A \int_{0}^{\tau-\xi} S(s) f(\xi) d s d \xi=\int_{0}^{\tau}[C(\tau-\xi) f(\xi)-f(\xi)] d \xi,
$$

which shows (b). Let $x^{*} \in D\left(A^{*}\right)$. Using the definition of $S(t)$, we have that the operator map $S(\cdot)$ is continuous for the norm of operators. This implies that the map $S(\cdot)^{*}$ is also continuous for the norm of operators. Combining with assertion (a) gives

$$
\begin{aligned}
\left\langle x, C(t)^{*} x^{*}-x^{*}\right\rangle=\left\langle C(t) x-x, x^{*}\right\rangle & =\left\langle A \int_{0}^{t} S(\xi) x d \xi, x^{*}\right\rangle \\
& =\int_{0}^{t}\left\langle S(\xi) x, A^{*} x^{*}\right\rangle d \xi=\int_{0}^{t}\left\langle x, S(\xi)^{*} A^{*} x^{*}\right\rangle d \xi
\end{aligned}
$$

for all $t \in \mathbb{R}$ and $x \in X$. Therefore, we have $C(t)^{*} x^{*}-x^{*}=\int_{0}^{t} S(\xi)^{*} A^{*} x^{*} d \xi$ which establishes (c). Similarly, it follows from (b) that

$$
\int_{0}^{t}\left\langle C(t-s) f(s), x^{\prime \prime}(t)\right\rangle d s=\int_{0}^{t}\left\langle f(s), x^{*}\right\rangle d s+\int_{0}^{t}\left\langle\int_{0}^{s} S(s-\xi) f(\xi) d \xi, A^{*} x^{*}\right\rangle d s
$$

and this equality yields assertion (d).

\section{A generation result}

In this section $A: D(A) \subseteq X \rightarrow X$ denotes a closed linear operator with dense domain. We begin with the concept of a weak solution.

Definition 2.1. A continuous function $u: I \rightarrow X$ is said to be a weak solution of the abstract Cauchy problem (1.1)-(1.2) if for each $x^{*} \in D\left(A^{*}\right)$ the function $\left\langle u(\cdot), x^{*}\right\rangle$ is continuously differentiable, the function $\frac{d}{d t}\left\langle u(t), x^{*}\right\rangle$ is absolutely continuous and the following conditions hold:

(i) $\frac{d^{2}}{d t^{2}}\left\langle u(t), x^{*}\right\rangle=\left\langle u(t), A^{*} x^{*}\right\rangle+\left\langle f(t), x^{*}\right\rangle$ a.e.;

(ii) $\left\langle u(0), x^{*}\right\rangle=\left\langle x_{0}, x^{*}\right\rangle$;

(iii) $\left.\frac{d}{d t}\left\langle u(t), x^{*}\right\rangle\right|_{t=0}=\left\langle x_{1}, x^{*}\right\rangle$. 
Remark 2.2. Since $D\left(A^{*}\right)$ is $w^{*}$-dense in $X^{*}$ ( [7, Theorem II.2.11]), it follows from (ii) above that $u(0)=x_{0}$. Similarly, if $u$ is a differentiable weak solution, it follows from (iii) that $u^{\prime}(0)=x_{1}$.

The following properties will be used to establish Theorem 2.5.

Lemma 2.3 ([7, Lemma VI.1.4]). Let $A$ be a closed linear operator with dense domain and let $x, y \in X$. If $\left\langle y, x^{*}\right\rangle=\left\langle x, A^{*} x^{*}\right\rangle$, for all $x^{*} \in D\left(A^{*}\right)$, then $x \in D(A)$ and $A x=y$.

In the next result we use the terminology of [5].

Lemma 2.4. Let $C:[-\tau, \tau] \rightarrow \mathcal{L}(X), \tau>0$, be a strongly continuous mapping such that $C(0)=I$ and the $D$ 'Alembert functional equation

$$
C(t+s)+C(t-s)=2 C(s) C(t), \quad-\frac{\tau}{2} \leq s, t \leq \frac{\tau}{2},
$$

is fulfilled. Then $C$ has a unique extension to $\mathbb{R}$ as a strongly continuous cosine function of operators on $X$.

Proof. We define the operator $A$ as in [5, Theorem II.3.1]. From the construction carried out in the proof of [5, Theorem II.3.1] we conclude that the Cauchy problem for the equation

$$
x^{\prime \prime}(t)=A x(t)
$$

is well posed in $\left[0, \frac{\tau}{2}\right]$. Since the equation (2.1) is autonomous, the Cauchy problem for $(2.1)$ is well posed in $[0, \infty)$. The assertion is now a consequence of [5, Theorem II.1.1].

We are now ready to state the main result of this section.

Theorem 2.5. Let $A$ be a closed linear operator with dense domain. Then the $A C P(1.1)-(1.2)$ has a unique weak solution for each $x_{0}, x_{1} \in X$ and each $f \in \mathcal{L}^{1}(I, X)$ if, and only if, $A$ is the infinitesimal generator of a strongly continuous cosine function of linear operators $C$. In this case, the weak solution coincides with the mild solution u given by (1.3) and

$$
\frac{d}{d t}\left\langle u(t), x^{*}\right\rangle=\left\langle S(t) x_{0}, A^{*} x^{*}\right\rangle+\left\langle C(t) x_{1}, x^{*}\right\rangle+\int_{0}^{t}\left\langle C(t-s) f(s), x^{*}\right\rangle d s
$$

holds for each $x^{*} \in D\left(A^{*}\right)$ and $0 \leq t \leq a$.

Proof. We assume initially that $A$ generates a strongly continuous cosine function of operators $C(t)$. We shall show that the mild solution $u$ given by (1.3) is also a weak solution. In fact, for each $x^{*} \in D\left(A^{*}\right)$ we have that

$$
\begin{aligned}
\left\langle u(t), x^{*}\right\rangle & =\left\langle C(t) x_{0}, x^{*}\right\rangle+\left\langle S(t) x_{1}, x^{*}\right\rangle+\int_{0}^{t}\left\langle S(t-s) f(s), x^{*}\right\rangle d s \\
& =\left\langle x_{0}, C(t)^{*} x^{*}\right\rangle+\left\langle S(t) x_{1}, x^{*}\right\rangle+\int_{0}^{t}\left\langle S(t-s) f(s), x^{*}\right\rangle d s .
\end{aligned}
$$


Applying Lemma 1.1(c) to the first term on the right hand side of the above expression, and using the properties of the convolution $S(\cdot) * f(\cdot)$ mentioned in the Introduction, we obtain that $\left\langle u(\cdot), x^{*}\right\rangle$ is continuously differentiable and (2.2) holds. Similarly, applying now Lemma 1.1(c) and (d) to the right hand side of (2.2), we infer that

$$
\begin{aligned}
\frac{d^{2}}{d t^{2}}\left\langle u(t), x^{*}\right\rangle= & \left\langle C(t) x_{0}, A^{*} x^{*}\right\rangle+\left\langle S(t) x_{1}, A^{*} x^{*}\right\rangle \\
& +\left\langle f(t), x^{*}\right\rangle+\int_{0}^{t}\left\langle S(t-s) f(s), A^{*} x^{*}\right\rangle d s, \text { a.e. } \\
= & \left\langle u(t), A^{*} x^{*}\right\rangle+\left\langle f(t), x^{*}\right\rangle, \text { a.e. }
\end{aligned}
$$

Since the conditions (ii) and (iii) of Definition 2.1 are immediate consequences of expressions (1.3) and (2.2), this completes the proof that $u$ is a weak solution. It only remains to show that $u$ is the unique weak solution. Utilizing the linearity of $A$ and the Definition 2.1, it follows that the difference of two weak solutions of the ACP (1.1)-(1.2) is a weak solution of the ACP problem

$$
\begin{aligned}
x^{\prime \prime}(t) & =A x(t), \quad t \in I=[0, a], \\
x(0) & =0, \quad x^{\prime}(0)=0 .
\end{aligned}
$$

Thus, in order to finish the proof, it is sufficient to show that a weak solution $x(\cdot)$ of (2.3)-(2.4) is $x=0$. To this end, we set $x^{*} \in D\left(A^{*}\right)$ and define the function $h(t)=\left\langle x(t), x^{*}\right\rangle$. Clearly, $h(0)=h^{\prime}(0)=0$ and $h^{\prime \prime}(t)=\left\langle x(t), A^{*} x^{*}\right\rangle$, a.e. Hence we infer that $h(t)=\left\langle\int_{0}^{t} \int_{0}^{s} x(\xi) d \xi d s, A^{*} x^{*}\right\rangle$. Setting $z(t)=\int_{0}^{t} \int_{0}^{s} x(\xi) d \xi d s$, we have that $h(t)=\left\langle x(t), x^{*}\right\rangle=\left\langle z(t), A^{*} x^{*}\right\rangle$. In view of that this property is verified for all $x^{*} \in D\left(A^{*}\right)$, Lemma 2.3 implies that $z(t) \in D(A)$ and $A z(t)=$ $x(t)$. Also, since $x(\cdot)$ is continuous, $z^{\prime \prime}(t)=x(t)=A z(t)$ and $z(0)=z^{\prime}(0)=0$. Applying the properties of the second order ACP mentioned in the Introduction, we deduce that $z(t)=C(t) z(0)+S(t) z^{\prime}(0)=0$ and, therefore $x(t)=0$.

Conversely, we assume now the existence of weak solutions for the problem (1.1)-(1.2). Let $u\left(t, x_{0}\right)$ be the weak solution of (1.1)-(1.2) corresponding to $x_{1}=0$ and $f=0$. For $0 \leq t \leq a$ we define the map $C(t): X \rightarrow X$ by

$$
C(t) x=u(t, x)
$$

It is clear from the Remark 2.2 that $C(0)=I$. Moreover, a standard argument using the uniqueness of the weak solutions allows us to conclude that $C(t)$ is a linear map. Furthermore, since $u(\cdot, x)$ is continuous the map $C(\cdot)$ is strongly continuous. We divide the rest of the proof into several steps.

Step 1. For each $0 \leq t \leq a$, the linear map $C(t)$ is bounded.

We define the mapping $V: X \rightarrow C(I, X), x \rightarrow C(\cdot) x$. From our preceding statements we have that $V$ is well defined with values in $C(I, X)$ and also $V$ is a 
linear map. Moreover, $V$ is a closed linear map. To put in evidence this fact, we take a sequence $\left(x_{n}\right)_{n}$ in $X$ such that $x_{n} \rightarrow x$ and $u\left(\cdot, x_{n}\right)=C(\cdot) x_{n} \rightarrow v(\cdot)$, as $n \rightarrow \infty$. This implies that $v$ is continuous and $\left\langle u\left(t, x_{n}\right), x^{*}\right\rangle \rightarrow\left\langle v(t), x^{*}\right\rangle, n \rightarrow$ $\infty$, uniformly for $t \in I$ and for every $x^{*} \in X^{*}$. This shows that

$$
\frac{d^{2}}{d t^{2}}\left\langle u\left(t, x_{n}\right), x^{*}\right\rangle=\left\langle u\left(t, x_{n}\right), A^{*} x^{*}\right\rangle, \text { a.e. }
$$

converges to $\left\langle v(t), A^{*} x^{*}\right\rangle, n \rightarrow \infty$, uniformly for $t \in I$ and for all $x^{*} \in D\left(A^{*}\right)$. By successive integration we get

$$
\left\langle u\left(t, x_{n}\right), x^{*}\right\rangle=\left\langle x_{n}, x^{*}\right\rangle+\int_{0}^{t} \int_{0}^{s}\left\langle u\left(\xi, x_{n}\right), A^{*} x^{*}\right\rangle d \xi d s,
$$

and taking limit as $n \rightarrow \infty$, we can write

$$
\left\langle v(t), x^{*}\right\rangle=\left\langle x, x^{*}\right\rangle+\int_{0}^{t} \int_{0}^{s}\left\langle v(\xi), A^{*} x^{*}\right\rangle d \xi d s,
$$

which implies that $v$ is a weak solution of (1.1)-(1.2) corresponding to $x_{0}=$ $x, x_{1}=0$ and $f=0$. Therefore, $v=V x$ and $V$ is a closed linear operator. From the closed graph theorem it follows that $V$ is a bounded operator. Thus, $\|C(t) x\| \leq\|V x\| \leq\|V\|\|x\|, t \in I$, which shows that $C(t)$ is also a bounded operator.

Step 2. The map $C: I \rightarrow \mathcal{L}(X)$ can be extended to $\mathbb{R}$ as a strongly continuous cosine function of operators.

We define $C(-t) x=C(t) x$, for $t \in I$ and for every $x \in X$. Let $x^{*} \in D\left(A^{*}\right)$. In view of that the right derivative of $\left\langle C(t) x, x^{*}\right\rangle$ at $t=0$ is equal to zero, it follows that the function $\left\langle C(t) x, x^{*}\right\rangle$ is continuously differentiable, its derivative is absolutely continuous and $\frac{d^{2}}{d t^{2}}\left\langle C(t) x, x^{*}\right\rangle=\left\langle C(t) x, A^{*} x^{*}\right\rangle$, for all $t \in[-a, a]$. Now, for each $t \in I$ we define the functions $v(s)=C(t+s) x+C(t-s) x$ and $w(s)=2 C(s) C(t) x$, for $s \in I$. Using the previous remarks, we obtain easily that both $v$ as $w$ are weak solutions of problem (1.1)-(1.2) corresponding to $x_{0}=2 C(t) x, x_{1}=0$ and $f=0$. Consequently, $v=w$, which shows that $C(\cdot)$ verifies the D'Alembert functional equation on $I$. The assertion is now consequence of Lemma 2.4. Next we utilize the symbol $C$ to denote this cosine function.

Step 3. The operator $A$ is the infinitesimal generator of $C$.

Let $A_{0}$ be the infinitesimal generator of $C$. Initially we prove that $A$ is an extension of $A_{0}$. Let $x \in D\left(A_{0}\right)$ and $x^{*} \in D\left(A^{*}\right)$. We define the function $h(t)=\left\langle C(t) x, x^{*}\right\rangle$. Then, combining the properties of cosine functions and Definition 2.1, we obtain

$$
h^{\prime \prime}(t)=\left\langle A_{0} C(t) x, x^{*}\right\rangle=\left\langle C(t) A_{0} x, x^{*}\right\rangle=\left\langle C(t) x, A^{*} x^{*}\right\rangle \text {, a.e. }
$$


Since the functions on the right hand side of the above expressions are continuous, we infer that the preceding equality holds for all $t \in I$. In particular, for $t=0$ we infer that $\left\langle A_{0} x, x^{*}\right\rangle=\left\langle x, A^{*} x^{*}\right\rangle$ and applying Lemma 2.3 we deduce that $x \in D(A)$ and $A x=A_{0} x$.

On the other hand, let $x \in X$ and $x^{*} \in D\left(A^{*}\right)$. Since $\frac{d^{2}}{d t^{2}}\left\langle C(t) x, x^{*}\right\rangle=$ $\left\langle C(t) x, A^{*} x^{*}\right\rangle$ by successive integration, we get

$$
\left\langle C(t) x-x, x^{*}\right\rangle=\left\langle\int_{0}^{t} \int_{0}^{s} C(\xi) x d \xi d s, A^{*} x^{*}\right\rangle .
$$

From Lemma 2.3, we conclude that $\int_{0}^{t} \int_{0}^{s} C(\xi) x d \xi d s \in D(A)$ and

$$
A \int_{0}^{t} \int_{0}^{s} C(\xi) x d \xi d s=C(t) x-x .
$$

Assume now that $x \in D(A)$. Applying the preceding result to $A x$ instead of $x$ we can assert that $\int_{0}^{t} \int_{0}^{s} C(\xi) A x d \xi d s \in D(A)$ and

$$
A \int_{0}^{t} \int_{0}^{s} C(\xi) A x d \xi d s=C(t) A x-A x .
$$

We define the function

$$
z(t)=\int_{0}^{t} \int_{0}^{s} C(\xi) A x d \xi d s-C(t) x+x, \quad t \geq 0 .
$$

Then the following properties hold:

(i) Clearly $z(\cdot)$ is continuous and $z(0)=0$.

(ii) It follows from (2.5) that for each $x^{*} \in D\left(A^{*}\right)$, the function $\left\langle z(\cdot), x^{*}\right\rangle$ is continuously differentiable and

$$
\frac{d}{d t}\left\langle z(t), x^{*}\right\rangle=\left\langle\int_{0}^{t} C(\xi) A x d \xi, x^{*}\right\rangle-\left\langle\int_{0}^{t} C(\xi) x d \xi, A^{*} x^{*}\right\rangle
$$

is continuous and $\left.\frac{d}{d t}\left\langle z(t), x^{*}\right\rangle\right|_{t=0}=0$.

(iii) Combining the assertion in (ii), equality (2.6) and the definition of $z$, we have

$$
\begin{aligned}
\frac{d^{2}}{d t^{2}}\left\langle z(t), x^{*}\right\rangle & =\left\langle C(t) A x, x^{*}\right\rangle-\left\langle C(t) x, A^{*} x^{*}\right\rangle \\
& =\left\langle A \int_{0}^{t} \int_{0}^{s} C(\xi) A x d \xi d s+A x, x^{*}\right\rangle-\left\langle C(t) x, A^{*} x^{*}\right\rangle \\
& =\left\langle z(t), A^{*} x^{*}\right\rangle .
\end{aligned}
$$


From (i), (ii) and (iii), we conclude that $z$ is a weak solution of problem (2.3)(2.4). As consequence of the uniqueness of weak solutions we obtain that $z(t)=0$. This yields $\int_{0}^{t} \int_{0}^{s} C(\xi) A x d \xi d s=C(t) x-x, t \geq 0$. This implies that

$$
2 \frac{C(t) x-x}{t^{2}}=\frac{2}{t^{2}} \int_{0}^{t} \int_{0}^{s} C(\xi) A x d \xi d s \rightarrow A x, \quad t \rightarrow 0^{+},
$$

Hence $x \in D\left(A_{0}\right)$ and $A_{0} x=A x$. This completes the proof.

\section{Second order abstract control systems}

In the remainder of this work $A$ denotes the infinitesimal generator of a strongly continuous cosine function of linear operators $C$ on the Banach space $X$. Furthermore, henceforth we assume that $X$ is a real Banach space. Certainly, if $X$ is a complex Banach space, our results are applicable to the underlying real space.

In this section, we are concerned with second order linear control systems that can be modeled by the equation

$$
x^{\prime \prime}(t)=A x(t)+B u(t),
$$

with states in $x(t) \in X$, controls $u(t)$ in a Banach space $U$ and where the control action $B: U \rightarrow X$ is a bounded linear operator. Both the exact and the approximate controllability of these systems have been studied by several authors. Directly related to systems modeled by equation (3.1) we mention $[6,10,21-23]$, and there is also an extensive literature related to functional systems $[16,17]$. Roughly speaking, the exact controllable systems are rather scarce, while they usually are approximately controllable. In this section, we study a concept, introduced by Baccioti in [3] and studied in [9] for abstract first order systems, similar but weaker than the controllability, which is called self-accessibility.

We consider as admissible trajectories of (3.1) in the time domain $I=[0, \tau]$ the mild solutions corresponding to control functions $u \in \mathcal{L}^{\infty}(I, U)$ and initial conditions $x(0)=x_{0}$ and $x^{\prime}(0)=x_{1}$. Consequently, the admissible trajectories of system (3.1) are given by

$$
x\left(t ; x_{0}, x_{1}, u\right)=C(t) x_{0}+S(t) x_{1}+\int_{0}^{t} S(t-s) B u(s) d s, \quad 0 \leq t \leq \tau,
$$

and it follows from Section 2 that the following relations hold:

$$
\begin{aligned}
\left\langle x\left(t ; x_{0}, x_{1}, u\right), x^{*}\right\rangle & =\left\langle C(t) x_{0}+S(t) x_{1}+\int_{0}^{t} S(t-s) B u(s) d s, x^{*}\right\rangle \\
\frac{d}{d t}\left\langle x\left(t ; x_{0}, x_{1}, u\right), x^{*}\right\rangle & =\left\langle S(t) x_{0}, A^{*} x^{*}\right\rangle+\left\langle C(t) x_{1}+\int_{0}^{t} C(t-s) B u(s) d s, x^{*}\right\rangle,
\end{aligned}
$$

for every $x^{*} \in D\left(A^{*}\right)$. These expressions lead to the following definition. 
Definition 3.1. The pair $\left(x_{0}, x_{1}\right)$ is said to be self-accessible on $I$ if there is $u \in \mathcal{L}^{\infty}(I, U)$ such that

$$
\begin{aligned}
& \left\langle x_{0}, x^{*}\right\rangle=\left\langle C(\tau) x_{0}+S(\tau) x_{1}+\int_{0}^{\tau} S(\tau-s) B u(s) d s, x^{*}\right\rangle \\
& \left\langle x_{1}, x^{*}\right\rangle=\left\langle S(\tau) x_{0}, A^{*} x^{*}\right\rangle+\left\langle C(\tau) x_{1}+\int_{0}^{\tau} C(\tau-s) B u(s) d s, x^{*}\right\rangle,
\end{aligned}
$$

for all $x^{*} \in D\left(A^{*}\right)$.

We have used expressions (3.3) and (3.4) to define a self-accessible state $\left(x_{0}, x_{1}\right)$ in order to avoid some a priori conditions on $x_{0}$ or $x_{1}$. Nevertheless, we can establish the following property.

Remark 3.2. If $\left(x_{0}, x_{1}\right)$ is self-accessible on $I$, it follows from (3.4) that

$$
\left\langle S(\tau) x_{0}, A^{*} x^{*}\right\rangle=\left\langle x_{1}-C(\tau) x_{1}-\int_{0}^{\tau} C(\tau-s) B u(s) d s, x^{*}\right\rangle,
$$

for all $x^{*} \in D\left(A^{*}\right)$. As consequence of Lemma 2.3, we derive that $S(\tau) x_{0} \in$ $D(A)$ and that

$$
A S(\tau) x_{0}=x_{1}-C(\tau) x_{1}-\int_{0}^{\tau} C(\tau-s) B u(s) d s .
$$

Combining this property with (3.3) and using the fact that $D\left(A^{*}\right)$ is $w^{*}$-dense in $X^{*}$ we obtain that $\left(x_{0}, x_{1}\right)$ is self-accessible on $I$ if, and only if, $S(\tau) x_{0} \in D(A)$ and there is $u \in \mathcal{L}^{\infty}(I, U)$ such that

$$
\begin{aligned}
& x_{0}=C(\tau) x_{0}+S(\tau) x_{1}+\int_{0}^{\tau} S(\tau-s) B u(s) d s \\
& x_{1}=A S(\tau) x_{0}+C(\tau) x_{1}+\int_{0}^{\tau} C(\tau-s) B u(s) d s .
\end{aligned}
$$

In this case, to abbreviate the terminology, we say that $x\left(\cdot, x_{0}, x_{1}, u\right)$ is a selfaccessible trajectory.

This motivates to introduce the space $E_{\tau}=\{x \in X: S(\tau) x \in D(A)\}$. Clearly, from the properties mentioned in the Introduction, $D(A) \subseteq E \subseteq E_{\tau}$.

Related to the concept of self-accessible states, we say that $x_{0} \in X$ is stationary on $I$ if there exists a control function $u \in \mathcal{L}^{\infty}(I, X)$ such that $x\left(t ; x_{0}, 0, u\right)=x_{0}$, for every $0 \leq t \leq \tau$ and we introduce the subspace $F=$ $\{x \in D(A): A x \in \mathcal{R}(B)\}$. Clearly, every $x_{0} \in F$ is stationary. In fact, if $A x_{0}+B u=0$ and we chose the constant control $u(t)=u$, then from Lemma 1.1 it follows that $x\left(t ; x_{0}, 0, u\right)=x_{0}$, for all $0 \leq t \leq \tau$.

We next show that the integral of a self-accessible trajectory is included in $F$. 
Proposition 3.3. If $x=x\left(\cdot, x_{0}, x_{1}, u\right)$ is a self-accessible trajectory of the control system (3.1), then $\int_{0}^{\tau} x(s) d s \in F$.

Proof. From (3.2) we can write

$$
\int_{0}^{\tau} x(s) d s=S(\tau) x_{0}+\int_{0}^{\tau} S(t) x_{1} d t+\int_{0}^{\tau} \int_{0}^{t} S(t-\xi) B u(\xi) d \xi d t .
$$

The first term on the right hand side belongs to $D(A)$ by Remark 3.2 while the second and third term on the right hand side belong to $D(A)$ by Lemma 1.1. Moreover, again applying Lemma 1.1 and combining with (3.6) we get that

$$
\begin{aligned}
A \int_{0}^{\tau} x(s) d s & =A S(\tau) x_{0}+(C(\tau)-I) x_{1}+\int_{0}^{\tau}[C(\tau-s)-I] B u(s) d s \\
& =-B \int_{0}^{\tau} u(s) d s
\end{aligned}
$$

which establishes the assertion.

Proposition 3.3 yields the following geometric property of self-accessible trajectories.

Theorem 3.4. Let $x=x\left(\cdot, x_{0}, x_{1}, u\right)$ be a self-accessible trajectory of the control system (3.1) on I, then

$$
\max _{0 \leq t \leq \tau}\left\|x_{0}-x(t)\right\| \geq d\left(x_{0}, F\right)
$$

Proof. We set $y=\frac{1}{\tau} \int_{0}^{\tau} x(t) d t$. From Proposition 3.3 we have that $y \in F$. Hence

$$
d\left(x_{0}, F\right) \leq\left\|x_{0}-y\right\|=\frac{1}{\tau}\left\|\int_{0}^{\tau}\left(x_{0}-x(t)\right) d t\right\| \leq \max _{0 \leq t \leq \tau}\left\|x_{0}-x(t)\right\| .
$$

The following consequence is immediate.

Corollary 3.5. Let $x_{0}$ be stationary on $I$. Then $x_{0} \in \bar{F}$.

In general, the subspace $F$ is not closed and, as a matter of fact, there are elements $x \in \bar{F}$ which are not stationary. As a simple example we can take $A=I, U=X$ and $B: X \rightarrow X$ a compact linear operator with dense range. Clearly, $F=\mathcal{R}(B)$ is dense in $X$. On the other hand, we define the operator $\Lambda: \mathcal{L}^{\infty}(I, X) \rightarrow X$ by the expression

$$
\Lambda_{1}(u)=\int_{0}^{\tau} S(\tau-s) B u(s) d s
$$


Then $\Lambda_{1}$ is a compact operator ([10]). Since $C(t)=\cosh t I$, for an stationary element $x \in X$, we can write $(1-\cosh \tau) x=\Lambda_{1}(u)$, for some $u \in \mathcal{L}^{\infty}(I, X)$. This shows that the stationary elements of $X$ are included in $\mathcal{R}\left(\Lambda_{1}\right) \neq X=\bar{F}$.

Certainly, if $A$ is a bounded operator and $\mathcal{R}(B)$ is a closed subspace, then $F$ is closed.

As a further application of Theorem 3.4 we can obtain a property of periodic cosine functions. The periodic cosine functions have been studied by several authors. In particular, from the characterization established in [15], we infer that if $C(\cdot)$ is $\tau$-periodic and $\operatorname{ker}(A)=\{0\}$, then $S(\tau)=0$.

Corollary 3.6. Assume that the cosine function $C$ is $\tau$-periodic and that $A$ is injective. Then, for every $x \in X$,

$$
\max _{0 \leq t \leq \tau}\|C(t) x-x\| \geq\|x\|
$$

Proof. We consider the control system (3.1) with $B=0$. Clearly, $(x, 0)$ is selfaccessible on the interval $[0, \tau]$, and since $F=\{0\}$, the assertion is a consequence of Theorem 3.4 .

We return to the study of control system (3.1) on the interval $[0, \tau]$. We define the maps $\Lambda_{2}: \mathcal{L}^{\infty}(I, X) \rightarrow X$ and $\Lambda: \mathcal{L}^{\infty}(I, X) \rightarrow X \times X$ by the expressions

$$
\Lambda_{2}(u)=\int_{0}^{\tau} C(\tau-s) B u(s) d s
$$

and $\Lambda=\left(\Lambda_{1}, \Lambda_{2}\right)$. It follows from (3.5), (3.6) and the definition of the group $G(t)$ that if $\left(x_{0}, x_{1}\right) \in E \times X$ is self-accessible, then

$$
(I-G(\tau))\left[\begin{array}{l}
x_{0} \\
x_{1}
\end{array}\right] \subseteq \mathcal{R}(\Lambda)
$$

We will denote by $P_{\tau}$ the space consisting of self-accessible vectors $\left(x_{0}, x_{1}\right) \in$ $E \times X$. System (3.1) is said self-accessible on $[0, \tau]$ if $P_{\tau}=E \times X$. In general, this property is rarely satisfied for infinite dimensional systems. To justify this assertion, we state the following.

Proposition 3.7. Assume that $B$ is a compact map and that there is an infinitedimensional closed subspace $Z_{\tau}$ of $E \times X$ such that $Z_{\tau} \subseteq \mathcal{R}(I-G(\tau))$. Then $P_{\tau} \neq E \times X$.

Proof. It follows from [10] that $\Lambda$ is a compact map. If $P_{\tau}=E \times X$, from the preceding remark we have that $\mathcal{R}(I-G(\tau)) \subseteq \mathcal{R}(\Lambda)$, which implies that $Z_{\tau} \subseteq \mathcal{R}(\Lambda)$, but this is a contradiction by the open mapping theorem. This proves the assertion. 
For real control systems the control space $U$ has finite dimension and the operator $B$ is compact. Consequently, for large classes of systems $P_{\tau} \neq E \times X$. We illustrate the criterion established in Proposition 3.7 with an application.

Example 3.8. Consider the controlled wave equation

$$
\begin{aligned}
\frac{\partial^{2} w(t, \xi)}{\partial t^{2}} & =\frac{\partial^{2} w(t, \xi)}{\partial \xi^{2}}+\sum_{i=1}^{m} b_{i}(\xi) u_{i}(t), \quad t>0,0<\xi<\pi \\
w(t, 0) & =w(t, \pi)=0 \\
w(0, \xi) & =w_{0}(\xi), \quad \frac{\partial w(t, \xi)}{\partial t}=w_{1}(\xi) .
\end{aligned}
$$

This system can be modeled in the form (3.1) on $X=L^{2}([0, \pi])$. The operator $A$ is defined by

$$
(A z)(\xi)=\frac{d^{2} z(\xi)}{d \xi^{2}}
$$

with domain $D(A)=\left\{z \in X: z \in H^{2}([0, \pi]), z(0)=z(\pi)=0\right\}$. The spectrum of $A_{1}$ consists of eigenvalues $-n^{2}$ for $n \in \mathbb{N}$, with associated eigenvectors $\varphi_{n}(\xi)=$ $\left(\frac{2}{\pi}\right)^{\frac{1}{2}} \sin (n \xi)$. Furthermore, the set $\left\{\varphi_{n}: n \in \mathbb{N}\right\}$ is an orthonormal basis of $X$. In particular,

$$
A x=\sum_{n=1}^{\infty}-n^{2}\left\langle x, \varphi_{n}\right\rangle \varphi_{n} .
$$

for $x \in D(A)$ ( [20, Example 5.1], [13, p. 117]). Using (3.10), one easily verifies that the operators $C(t)$ defined by

$$
C(t) x=\sum_{n=1}^{\infty} \cos (n t)\left\langle x, \varphi_{n}\right\rangle \varphi_{n}, \quad t \in \mathbb{R}
$$

form a cosine function on $X$. We take $\tau=\pi$. Let $X_{0}$ be the closure of the subspace generated by the functions $\varphi_{2 n-1}$ for $n \in \mathbb{N}$. For $x \in X_{0}$, we obtain that $C(\tau) x=-x$ and $S(\tau) x=0$. Therefore,

$$
(I-G(\tau))\left[\begin{array}{l}
0 \\
x
\end{array}\right]=\left[\begin{array}{cc}
I-C(\tau) & -S(\tau) \\
-A S(\tau) & I-C(\tau)
\end{array}\right]\left[\begin{array}{l}
0 \\
x
\end{array}\right]=2\left[\begin{array}{l}
0 \\
x
\end{array}\right] .
$$

Hence, the subspace $Z_{\tau}=\{0\} \times X \subseteq \mathcal{R}(I-G(\tau))$. Assuming that $b_{i} \in X$ for $i=1, \ldots, m$, the operator $B: \mathbb{R}^{m} \rightarrow X$ is given by $B\left(u_{1}, \ldots, u_{m}\right)=\sum_{i=1}^{m} u_{i} b_{i}$. It is clear that $B$ is compact. By Proposition 3.7, system (3.7)-(3.9) is not self-accessible.

These results lead us to consider the weaker concept of approximate selfaccessibility. If $x=x\left(\cdot, x_{0}, x_{1}, u\right)$ is an admissible trajectory with $x_{0} \in E_{\tau}$, we shall denote

$$
y(\tau)=A S(\tau) x_{0}+C(\tau) x_{1}+\int_{0}^{\tau} C(t-s) B u(s) d s .
$$


Definition 3.9. Let $x_{0} \in E_{\tau}$. We say that $\left(x_{0}, x_{1}\right)$ is approximately selfaccessible on $I$ if for each $\varepsilon>0$, there exists an admissible trajectory $x=$ $x\left(\cdot, x_{0}, x_{1}, u\right)$ such that $\left\|x(\tau)-x_{0}\right\|+\left\|y(\tau)-x_{1}\right\| \leq \varepsilon$. In this case we say that $x(\cdot)$ is an admissible trajectory of $\varepsilon$-self-accessibility.

We introduce some additional notations. If $x^{*} \in D\left(A^{*}\right), A^{*} x^{*} \neq 0$, we denote by $P\left(x^{*}\right)$ the closed hyperplane formed by the elements $x \in X$ such that $\left\langle x, A^{*} x^{*}\right\rangle=0$. Moreover, $N\left(B^{*}\right)=\operatorname{ker}\left(B^{*}\right) \cap D\left(A^{*}\right)$. In the calculation that follows we use the well known property that the distance of a point $z$ to the hyperplane $P=\left\{x \in X:\left\langle x, y^{*}\right\rangle=0\right\}$ is given by $d(z, P)=\frac{\left|\left\langle z, y^{*}\right\rangle\right|}{\left\|y^{*}\right\|}$.

Theorem 3.10. Let $x_{0} \in E_{\tau}$ and let $x=x\left(\cdot, x_{0}, x_{1}, u\right)$ be an admissible trajectory of the control system (3.1) on I such that $\left\|x_{1}-y(\tau)\right\| \leq \delta$, for some $\delta>0$. If $x^{*} \in N\left(B^{*}\right)$ and $A^{*} x^{*} \neq 0$, then there is $s \in I$ such that

$$
d\left(x(s), P\left(x^{*}\right)\right) \leq \frac{\delta}{\tau} \frac{\left\|x^{*}\right\|}{\left\|A^{*} x^{*}\right\|} .
$$

In particular, if $x$ is a trajectory of self-accessibility, then there is $s \in I$ such that $x(s) \in P\left(x^{*}\right)$.

Proof. We define $h(t)=\left\langle x(t), x^{*}\right\rangle$. Since $B^{*} x^{*}=0$, it follows from the Section 2 that $h$ is continuously differentiable, $h^{\prime}$ is absolutely continuous and $h^{\prime \prime}(t)=$ $\left\langle x(t), A^{*} x^{*}\right\rangle$ is continuous. Hence, we have that $h^{\prime}$ is continuously differentiable. Moreover, $\left|h^{\prime}(0)-h^{\prime}(\tau)\right|=\left|\left\langle x_{1}-y(\tau), x^{*}\right\rangle\right| \leq \delta\left\|x^{*}\right\|$. From the mean value theorem we find that there exists $s \in I$ such that $\tau\left|\left\langle x(s), A^{*} x^{*}\right\rangle\right|=\left|h^{\prime \prime}(s)\right| \tau=$ $\left|h^{\prime}(0)-h^{\prime}(\tau)\right|$. Since $d\left(x(s), P\left(x^{*}\right)\right)=\frac{\left|\left\langle x(s), A^{*} x^{*}\right\rangle\right|}{\left\|A^{*} x^{*}\right\|}$, we obtain the first assertion. The second statement is an immediate consequence of this result by taking $\delta \rightarrow 0$.

We are able now to establish an extension of Theorem 3.4 for trajectories that have a property of approximate self-accessibility.

Theorem 3.11. Assume that $F^{\perp} \subseteq A^{*}\left(D\left(A^{*}\right) \cap \operatorname{ker}\left(B^{*}\right)\right)$. Let $x_{0} \in E_{\tau}$ such that $d\left(x_{0}, F\right)>0$. Then for each $\varepsilon>0$, there exists $\delta>0$ such that

$$
\max _{0 \leq t \leq \tau}\left\|x(t)-x_{0}\right\| \geq d\left(x_{0}, F\right)-\varepsilon
$$

for every admissible trajectory $x=x\left(\cdot, x_{0}, x_{1}, u\right)$ of the control system (3.1) on I such that $\left\|x_{1}-y(\tau)\right\| \leq \delta$.

Proof. We set $r=d\left(x_{0}, F\right)$. From the Hahn-Banach theorem, we infer that there exists a closed hyperplane $H$ in $X$ which contains $F$ and $d\left(x_{0}, H\right)=r$. Let $y^{*} \in X^{*}$ such that $H=\left\{x \in X:\left\langle x, y^{*}\right\rangle=0\right\}$. Since $y^{*} \in F^{\perp}$, using our hypothesis we can affirm that there exists $x^{*} \in N\left(B^{*}\right)$ such that $H=P\left(x^{*}\right)$. 
This implies that $A^{*} x^{*} \neq 0$ and we can select a constant $\delta>0$ such that $\delta \leq \varepsilon \tau \frac{\left\|A^{*} x^{*}\right\|}{\left\|x^{*}\right\|}$. It follows from Theorem 3.10 that there is $s \in I$ such that $d(x(s), H) \leq \varepsilon$. This implies that $\max _{0 \leq t \leq \tau}\left\|x(t)-x_{0}\right\| \geq\left\|x(s)-x_{0}\right\| \geq$ $\left|d\left(x_{0}, F\right)-d(x(s), F)\right|$, which completes the proof.

Remark 3.12. In [11, Theorem 3], we have shown that if $\mathcal{R}(A)+\mathcal{R}(B)$ is closed in $X$ and $F$ is included in a closed hyperplane $H$, then $H=P\left(x^{*}\right)$, for some $x^{*} \in$ $N\left(B^{*}\right)$. Consequently, we can change the condition $F^{\perp} \subseteq A^{*}\left(D\left(A^{*}\right) \cap \operatorname{ker}\left(B^{*}\right)\right)$ in Theorem 3.11 by the condition $\mathcal{R}(A)+\mathcal{R}(B)$ closed.

Acknowledgment. The authors thank the referee for his valuable comments and suggestions.

\section{References}

[1] Arendt, W., Batty, C., Hieber, M. and Neubrander, F., Vector-Valued Laplace Transforms and Cauchy Problems. Basel: Birkhäuser 2001.

[2] Baccioti, A., Auto-acessibilité par familles de champs de vecteurs (in French). Ricerche di Automatica 7 (1976), 189 - 197.

[3] Ball, J. M., Strongly continuous semigroups, weak solutions and the variation of constants formula. Proc. Amer. Math. Soc. 63 (1977)(2), 370 - 373.

[4] Bochenek, J., An abstract nonlinear second order differential equation. Ann. Polon. Math. 54 (1991)(2), 155 - 166.

[5] Fattorini, H. O., Second Order Linear Differential Equations in Banach Spaces. North-Holland Math. Studies 108. Amsterdam: North-Holland 1985.

[6] Fattorini, H. O., Controllability of higher order linear systems. In: Mathematical Theory of Control (Proc. Conf., Los Angeles 1967; eds.: A. V. Balakrishnan and L. W. Neustad) New York: Academic Press 1967, pp. $301-311$.

[7] Goldberg, S., Unbounded Linear Operators. New York: Dover 1966.

[8] Haase, M., The Functional Calculus for Sectorial Operators. Basel: Birkhäuser 2006.

[9] Henríquez, H. R., Auto-acessibilidade de sistemas de controle lineares em espacos de Banach (in Portuguese). Anais do $1^{\circ}$ Congresso Latino-Americáno de Automática (Campina Grande, Brasil, 1984; ed.: E. R. Cabral da Silva), Vol. III. Federal University of Paraíba 1984, pp. 860 - 865.

[10] Henríquez, H. R., On nonexact controllable systems. Internat. J. Control 42 (1985)(1), $71-83$.

[11] Henríquez, H. R., Castillo, G. and Rodriguez A., A geometric property of control systems with states in a Banach space. Systems Control Lett. 8 (1987)(3), $225-229$.

[12] Henríquez, H. R. and Vásquez, C. H., Differentiabilty of solutions of the second order abstract Cauchy problem. Semigroup Forum 64 (2002)(3), 472 - 488. 
[13] Hinrichsen, D. and Pritchard, A. J., Mathematical Systems Theory I. Berlin: Springer 2005.

[14] Kisyński, J., On cosine operator functions and one parameter group of operators. Studia Math. 44 (1972), 93 - 105.

[15] Lutz, D., Periodische operatorwertige Cosinusfunktionen (in German). Resultate Math. 4 (1981)(1), 75 - 83.

[16] McKibben, M. A., Approximate controllability for a class of abstract secondorder functional evolution equations. J. Optim. Theory Appl. 117 (2003)(2), $397-414$.

[17] Park, J. Y., Kwun, Y. C. and Lee, H. J., Controllability of second-order neutral functional differential inclusions in Banach spaces. J. Math. Anal. Appl. 285 (2003)(1), $37-49$.

[18] Travis, C. C. and Webb, G. F., Compactness, regularity, and uniform continuity properties of strongly continuous cosine families. Houston J. Math. 3 (1977)(4), $555-567$.

[19] Travis, C. C. and Webb, G. F., Cosine families and abstract nonlinear second order differential equations. Acta Math. Acad. Sci. Hungar. 32 (1978)(1-2), $75-96$.

[20] Travis, C. C. and Webb, G. F., Second order differential equations in Banach space. In: Nonlinear Equations in Abstract Spaces (Proc. Internat. Sympos., Univ. Texas, Arlington, Tex., 1977; ed.: V. Lakshmikantham). New York: Academic Press 1987, pp. 331 - 361.

[21] Triggiani, R., On the relationship between first and second order controllable systems in Banach spaces. In: Distributed Parameter Systems: Modeling and Identification (Proc. IFIP Working Conf., Rome, 1976; eds.: A. V. Balakrishnan and M. Thoma) Lecture Notes Control Inf. Sci. 1. Berlin: Springer 1978, pp. $370-393$.

[22] Triggiani, R., On the relationship between first and second order controllable systems in Banach spaces. SIAM J. Control Optim. 16 (1978)(6), 847 - 859.

[23] Tsujioka, K., Remarks on controllability of second order evolution equations in Hilbert spaces. SIAM J. Control 8 (1970), $90-99$.

Received November 27, 2006; revised September 25, 2007 